This item was submitted to Loughborough's Research Repository by the author.

Items in Figshare are protected by copyright, with all rights reserved, unless otherwise indicated.

\title{
Surface specific asperity model for prediction of friction in boundary and mixed regimes of lubrication
}

PLEASE CITE THE PUBLISHED VERSION

http://dx.doi.org/10.1007/s11012-016-0397-z

\section{PUBLISHER}

(C) The Authors. Published by Springer.

\section{VERSION}

VoR (Version of Record)

\section{PUBLISHER STATEMENT}

This work is made available according to the conditions of the Creative Commons Attribution 4.0 International (CC BY 4.0) licence. Full details of this licence are available at: http://creativecommons.org/licenses/ by/4.0/

\section{LICENCE}

CC BY-NC-ND 4.0

\section{REPOSITORY RECORD}

Leighton, Michael, Nicholas J. Morris, Ramin Rahmani, and Homer Rahnejat. 2019. "Surface Specific Asperity Model for Prediction of Friction in Boundary and Mixed Regimes of Lubrication”. figshare.

https://hdl.handle.net/2134/20745. 


\title{
Surface specific asperity model for prediction of friction in boundary and mixed regimes of lubrication
}

\author{
M. Leighton • N. Morris $\cdot$ R. Rahmani • \\ H. Rahnejat
}

Received: 27 July 2015/ Accepted: 18 February 2016

(C) The Author(s) 2016. This article is published with open access at Springerlink.com

where commonly used Gaussian distributions can lead to anomalous predictions.

Keywords Real rough surfaces $\cdot$ Contact load carrying capacity $\cdot$ Friction

\section{List of symbols}

$\tilde{\mathrm{A}} \quad$ Mean area of asperity contact in the apparent area of contact

$\mathcal{A} \quad$ Apparent area of contact

$a$ Acceleration of floating plate

$b \quad$ Strip face-width

c Lubricant rupture boundary

$d \quad$ Surface separation of mean centrelines

$d_{i} \quad$ Height above the centreline of a surface

$E^{\prime} \quad$ Composite modulus of elasticity

$f \quad$ Total friction

$f_{b} \quad$ Boundary friction

$f_{v} \quad$ Viscous friction

$\mathrm{F}_{5 / 2} \quad$ Statistical function for asperity load carrying capacity

$\mathrm{F}_{2} \quad$ Statistical function for asperity contact area

$h \quad$ Separation or film thickness

$h_{0} \quad$ Initial gap between surfaces

$h_{i n i} \quad$ Height of the highest peak relative to the mean centre-line of the surface

$m \quad$ Mass of floating plate

$L \quad$ Length of sliding strip

$p \quad$ Hydrodynamic pressure

$p_{\text {atm }} \quad$ Atmospheric pressure

$p_{\text {cav }}$ Cavitation pressure 
$P \quad$ Load carried by one asperity pair

$\tilde{P} \quad$ Mean asperity load carried for the apparent area of contact

$r \quad$ Radial distance between asperity tips

$R \quad$ Crown radius of the ring parabolic profile

$t \quad$ Time

$U \quad$ Sliding velocity

$w \quad$ Peak penetration

$w_{p} \quad$ Vertical interference between asperity tips

$W \quad$ Applied load on the strip (total contact load)

$W_{h y} \quad$ Hydrodynamic reaction

$\mathrm{z}_{i} \quad$ Topography height above the centreline of surfaces

Z Pressure-viscosity index

\section{Greek symbols}

$\alpha \quad$ Piezo-viscosity

$\beta \quad$ Asperity tip radius

$\delta \quad$ Surface deformation

$\eta \quad$ Lubricant dynamic viscosity

$\eta_{0} \quad$ Lubricant dynamic viscosity at ambient conditions

$\lambda \quad$ Stribeck parameter, $\lambda=h / \sigma$

$\xi \quad$ Asperity density per unit area

$\rho \quad$ Lubricant density

$\rho_{0} \quad$ Lubricant density at ambient conditions

$\varsigma \quad$ Coefficient of the boundary shear strength

$\phi \quad$ Probability distribution function

$\phi^{*} \quad$ Convoluted probability distribution function

$\sigma \quad$ Root mean square variation from mean surface centreline

$\tau_{0} \quad$ Eyring shear stress of the lubricant

$\Psi \quad$ Asperity tip curvatures

\section{Introduction}

A sufficiently thick low shear strength film of lubricant is usually desired to form in all contact conjunctions to carry the applied load and guard against the direct interaction of asperities on the opposing surfaces, thus reducing frictional losses. However, in practice this ideal situation is often not achieved, owing to many circumstances, such as stop-start or reciprocating motions, which affect the entrainment of the lubricant into the contact with relative motion of surfaces [1,2]. There may also be a lack of lubricant availability at the inlet to a conjunction as well as reverse and swirl flows there $[3,4]$. As the result, many contacts suffer from a lack of a coherent lubricant film where a proportion of applied load is carried by the ubiquitous asperities on the counterface surfaces. These interactions increase the generated friction, for example in the cases of piston-cylinder system at dead centre reversals [5-8] and cam-follower contact in the inlet reversal positions [9]. Various palliative actions are undertaken in order to mitigate these adverse effects within the mixed regime of lubrication, including the use of hard wear-resistant coatings. Another approach has been surface modifications such as cross-hatching of cylinder surfaces or texturing in order to entrap reservoirs of lubricant for instances of poor entraining motion. In order to opt for any method of palliation, it would be instructive to initially predict the extent of boundary interactions in an accurate manner.

Greenwood and Tripp [10] and Greenwood and Williamson [11] provided mathematical discourses for asperity interactions between pairs of rough surfaces for simplified asperity geometry and an assumed Gaussian distribution of peak heights. A further series of assumptions were made, most crucially an average asperity tip radius and an average indentation depth at given separations with the mutual approach of rough counterfaces. The method also accounted for the oblique interaction of any pair of opposing asperities. These are usually regarded as necessary assumptions to deal with the complex nature of rough surface topography. The Gaussian assumption made in Greenwood and Tripp [10] is not a prerequisite of the method. Consideration of typical rough surfaces suggests that a peak height distribution would have a mean value above the mid-line of the surface as the majority of the peaks would reside in the upper reaches of the surface. It is, therefore, advantageous to develop an asperity model which allows more representative peak height distributions to be utilised, particularly for lubricated contacts which were not discussed by Greenwood and Tripp [10].

Greenwood and Tripp [10] used a Gaussian distribution to approximate the probability of peak height interactions at a given surface separation; $\lambda$ where this is inversely proportional to $\sigma$ (root mean square; RMS) of the surface heights. As a result the mean of the peak height distribution is set to the same mean as the surface height distribution as a simplification for the proof of concept which, for engineering surfaces, tends to predict that the first surface interactions occur at a lower value of $\lambda$ than is otherwise the case. This 
results in an estimate of asperity load carrying capacity significantly below a real case at the upper reaches of the surface and a lower separation limit at which the mixed regime of lubrication is expected to commence. One way to deal with this shortcoming is to measure the mean peak height relative to the surface centre-line and shift the mean of the Gaussian distribution to this location. This requires the analysis of the surface and identification of the peak height distribution. Therefore, "the surface specific distribution" might as well be used for the rest of the analysis, thus improving the accuracy of surface representation with little extra effort. Furthermore, the Gaussian distribution used to describe the peak height distribution must have the same standard deviation as the actual peak height distribution, not the RMS roughness, $\sigma$, which is derived from the surface height distribution.

Greenwood and Williamson's model [11] was further developed to account for non-uniform radii of curvature of asperity peaks by Hisakado [12], and for elliptic paraboloid asperities by Bush et al. [13], as well as for anisotropic surfaces by McCool [14]. The Greenwood and Tripp model [10] was extended by Pullen and Williamson [15] to account for the plastic deformation of asperities and further improved by Cheng et al. [16] for an elasto-plastic model. A recent extension of the model for combined elasto-plastic and adhesion of asperities for fairly smooth surfaces, using fractal geometry was reported by Chong et al. [17]. Nevertheless, the original Greenwood and Tripp model [10] has been widely used in many applications [18-21].

\section{Background Theory}

It is initially assumed that the surfaces are replaced by an equivalent surface comprising a collection of hemispherical asperities of an average tip radius, $\beta$

Fig. 1 Geometry of asperity pair interaction
(Fig. 1) and with the same peak height distribution as the original surfaces themselves.

The asperity radii $\left(\beta_{1}\right.$ and $\left.\beta_{2}\right)$ and the radial separation $r$ allow $w_{p}$ (the peak height interference) to be related to $w$ (the asperity compression) this results in the load carrying capacity of the surface. For the interference of two spheres the asperity compression can be shown to be:

$w=\left\langle w_{p}-\frac{r^{2}}{2\left(\beta_{1}+\beta_{2}\right)}\right\rangle$

where, \langle\rangle is a "Macauley bracket". Let:

$\beta=\frac{\beta_{1}+\beta_{2}}{2}$

where:

$\beta_{1}=\frac{\sum_{i=1}^{n} \beta_{1 i}}{n}$

The average asperity density over both surfaces is $\xi$ and the apparent area of contact is $\mathcal{A}$. Therefore, the probable load supported by this representative equivalent surface at a given separation, $\tilde{P}(d)$ becomes:

$$
\begin{gathered}
\tilde{P}(d)=2 \pi \xi^{2} \mathcal{A} \int_{r} \int_{z_{1}} \int_{z_{2}} P(w, r) \phi\left(z_{1}\right) \phi\left(z_{2}\right) r \cdot d z_{2} . \\
d z_{1} \cdot d r
\end{gathered}
$$

where, $P(w, r)$ is the load carried by an asperity pair with misalignment $r$ and peak penetration of $w$. Equation (4) states that the total carried load is as the result of the load supported at a given penetration between all pairs of opposing asperities at any given surface height.

The surface specific contributions are the peak height distributions, $\phi\left(z_{1}\right)$ and $\phi\left(z_{2}\right)$, and the asperity peak density, $\xi$. Examples of measured surface height

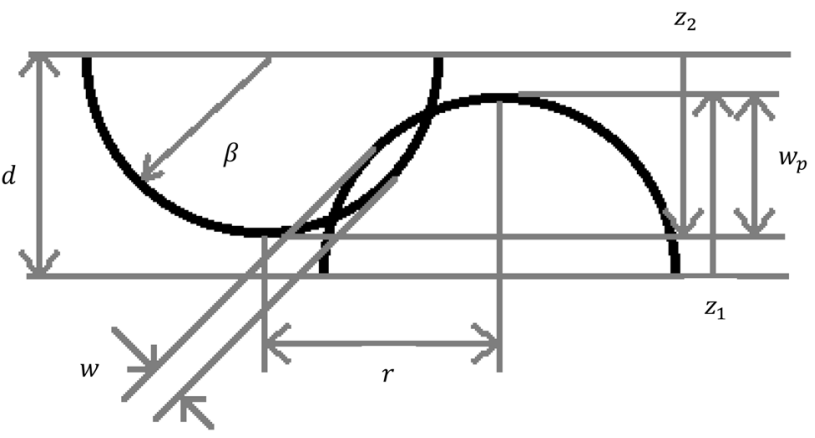


distributions can be seen in the work of Peklenik [22] who also discussed some of the key variations between measured and generated surface properties.

Combining the probabilities by taking the convolution of the asperity peak height distributions for counterface surfaces leads to:

$\phi^{*}(z)=\phi\left(z_{1}\right) * \phi\left(z_{2}\right)$

Using Hertzian theory to express the load carrying capacity as a function of the deformation (penetration) of two spheres in elastic contact with the effective composite elastic modulus, $E^{\prime}$ :

$P=\frac{4}{3} E^{\prime} \Psi^{\frac{1}{2}} W^{\frac{3}{2}}$

Where $\Psi$ is the composite curvatures for two contacting spheres defined as:

$\frac{1}{\Psi}=\frac{1}{\beta_{1}}+\frac{1}{\beta_{2}}$

Equation (4) now reduces to:

$\tilde{P}(d)=2 \pi \xi^{2} \mathcal{A} \int_{r} \int_{z}\left(\frac{4}{3}\right) E^{\prime} \Psi^{\frac{1}{2}} W^{\frac{3}{2}} \phi^{*}(Z) r . d z . d r$

With the inclusion of Eqs. (1) and (2) the equation for the probable asperity carried load at a given separation simplifies to:

$\tilde{P}(d)=2 \pi \xi^{2} \mathcal{A} \int_{r} \int_{z}\left(\frac{4}{3}\right) E^{\prime} \Psi^{\frac{1}{2}}\left\langle w_{p}-\frac{r^{2}}{4 \beta}\right\rangle^{\frac{3}{2}} \phi^{*}(Z) r . d z . d r$

Extracting the constants and setting the limits of integration, yields:

$$
\begin{aligned}
\tilde{P}(d)= & \frac{8}{3} \pi \xi^{2} E^{\prime} \Psi^{\frac{1}{2}} \mathcal{A} \int_{d}^{\infty} \phi^{*}(z) \cdot d z \\
& \int_{0}^{\infty}\left\langle w_{p}-\frac{r^{2}}{4 \beta}\right\rangle^{\frac{3}{2}} r \cdot d r \\
\tilde{P}(d)= & \frac{8}{3} \pi \xi^{2} E^{\prime} \Psi^{\frac{1}{2}} \mathcal{A} \int_{d}^{\infty} \phi^{*}(z) \cdot d z \\
& {\left[-\frac{4}{5} \beta\left\langle w_{p}-\frac{r^{2}}{4 \beta}\right\rangle^{\frac{5}{2}}\right]_{0}^{\infty} } \\
\tilde{P}(d)= & \frac{32}{15} \pi \xi^{2} E^{\prime} \Psi^{\frac{1}{2}} \beta \mathcal{A} \int_{d}^{\infty}\langle z-d\rangle^{\frac{5}{2}} \phi^{*}(z) \cdot d z
\end{aligned}
$$

Standardising the form of the probability distribution as $s=\sigma z$ :
$\tilde{P}(d)=\frac{32}{15} \pi \xi^{2} \Psi^{\frac{1}{2}} \beta \sigma^{\frac{5}{2}} E^{\prime} \mathcal{A} \int_{d / \sigma}^{\infty}\left(s-\frac{d}{\sigma}\right)^{\frac{5}{2}} \phi^{*}(s) \cdot d s$

Equation (13) can then be rearranged to:

$\tilde{P}(d)=\frac{32}{15} \pi \xi^{2} \Psi^{\frac{1}{2}} \beta \sigma^{\frac{5}{2}} E^{\prime} \mathcal{A} \mathrm{F}_{\frac{5}{2}}\left(\frac{d}{\sigma}\right)$

In a similar manner, the mean asperity contact area within the apparent contact area is found as:

$\tilde{A}(d)=2 \pi^{2}(\xi \sigma)^{2} \Psi \beta \mathcal{A} F_{2}(\lambda)$

Where the probability functions in Eqs. (14) and (15) are defined as:

$F_{n}(\lambda)=\int_{\lambda}^{\infty}(s-\lambda)^{n} \phi^{*}(s) \cdot d s$

It is of note that in the original theory proposed by Greenwood and Tripp [10] the radii on each surface are assumed to be equal and therefore:

$\Psi=\frac{\beta}{2}$

If Eq. (17) is true then Eqs. (14) and (15) respectively reduce to those given in [10] as

$\tilde{P}(d)=\frac{16 \sqrt{2}}{15} \pi(\xi \beta \sigma)^{2} E^{\prime} \sqrt{\frac{\sigma}{\beta}} \mathcal{A} \mathrm{F}_{\frac{5}{2}}\left(\frac{d}{\sigma}\right)$

$\tilde{A}(d)=\pi^{2}(\xi \beta \sigma)^{2} \mathcal{A} F_{2}(\lambda)$

The function, $F_{5 / 2}(\lambda)$, represents the statistical likelihood of interactions of deformed asperities at all separations as one surface is lowered onto the other. In order to improve the accuracy of the model for a specific pair of surfaces the specific parameter must be calculated. The parameters; $\xi, \beta$ and $\sigma$ can be readily determined by many commercially available metrology systems. Determination of the function $F_{5 / 2}(\lambda)$ from measured surface topography is more involved. As already noted, to correctly apply the assumption of a Gaussian distribution of peak heights, the standard deviation and mean of the peak heights are needed a priori. However, it is more representative to determine the peak height distributions of the surfaces through a simple extension of the approach highlighted above, using instead the surface specific data.

$F_{5 / 2}(\lambda)$ and the roughness parameter determine the probable number of asperities which are penetrated at 
a given surface separation and the extent of their deformation.

\section{Extension to measured surface data}

Measuring surface topography using a variety of measurement techniques yields an array of surface height data at discrete measured nodes. The discrete surface data is then used in a probabilistic model, such as that described above, with the frequency distributions required in the form of a histogram of each surface. The distribution is dependent on the size and resolution of the sampled area, as well as the surface itself.

Identifying the asperity summits and their heights leads to a discrete peak height distribution for each surface. The convolution of these two peak height distributions is then found, $\phi^{*}$, must conform to:

$\int_{-\infty}^{\infty} \phi^{*}(x) \cdot d x=1$

Then, the calculation of functions such as $F_{5 / 2}(\lambda)$ is a routine matter for any desired surface height through integration of the histogram columns residing above the $\lambda$ value being considered with a weighting as shown in Eq. (16).

The remaining surface specific parameters considered in this model are: $\xi, \beta, \Psi$ are and $\sigma$. The determination of $\beta$ and $\Psi$ not considered satisfactory techniques for measured surfaces. $\beta$ represents the mean radius of all the asperity pairs in contact at a given separation. For multi-scale surfaces, such as cross-hatched cylinder liners, the manufacturing process often comprises several stages. Honing creates a rough, isotropic surface with a negatively skewed surface height distribution by machining away the upper levels of the surface with successively smoother machining processes. This means that there is a variation in $\beta$ at different heights of the surface. At the upper levels there are likely to be smoother and more rounded asperities, whilst any lower peaks formed by the initial machining process and untouched by any subsequent operation would have a lower asperity radius (i.e. sharper peaks). As a result a variable $\beta$ value is encountered at different separations.

For the individual surfaces, $\beta_{i}(h)$ should be determined as the mean of all the asperity radii above a given height, $h$, from the centre-line of the topography as weighted by their heights since higher asperities have a greater probability of contacting the countersurface. Taking a double integral, the variable asperity radius can be determined from:

$\beta_{1}(h)=\int_{h}^{h_{\text {ini1 }}} \int_{x}^{h_{i n i 1}} \frac{\sum_{i=1}^{n} \beta_{1 i}(s)}{n} d s d x$

Subsequently, the mean of the same with any probability of contact at any given separation should be used. With the variation of the asperity radii of the individual counter surfaces determined, consideration of the combined effect from two surfaces in contact should then be considered. The two functions thus found for the individual surfaces cannot be convoluted in the same manner as the peak height distributions. It is necessary to know the height of the upper most asperity on each surface in order to determine the maximum separation at which a contact can be made. These peak asperity pairs represent the initial possible direct contact of the surfaces as the mean centre-lines are moved closer. They are designated as $h_{\text {ini1 }}$ and $h_{\text {ini2 }}$. A variable asperity radius distribution can then be found as:

$\beta(h)=\frac{\beta_{1}\left(h-h_{\text {ini2 }}\right)+\beta_{2}\left(h-h_{i n i 1}\right)}{2}$

Similarly, the variable asperity curvature $\Psi_{i}(h)$ can be determined from:

$$
\begin{aligned}
& \Psi_{1}(h)=\int_{h}^{h_{i n i 1}} \int_{x}^{h_{i n i 1}} \frac{\sum_{i=1}^{n} \frac{1}{\beta_{1 i}(s)}}{n} d s d x \\
& \Psi(h)=\frac{2}{\Psi_{1}\left(h-h_{\text {ini } 2}\right)+\Psi_{2}\left(h-h_{\text {ini } 1}\right)}
\end{aligned}
$$

\section{Results for measured surfaces}

Measured data from real surfaces is used in order to determine a more realistic $F_{5 / 2}(\lambda)$ function. The surfaces considered in this study are those of a flat plate with a skewed surface height distribution and a surface finish similar to that of a plateau-honed cylinder liner (surface 1 as shown in Fig. 2a) and a corresponding flat surface with an approximately Gaussian surface height distribution and low RMS 
Fig. 2 Images of surface topography using an Alicona Infinite Focus Microscope at $\times 50$ optical magnification applying the focus variation technique: $284 \times 216 \mu \mathrm{m}$. a Surface 1 . b Surface 2

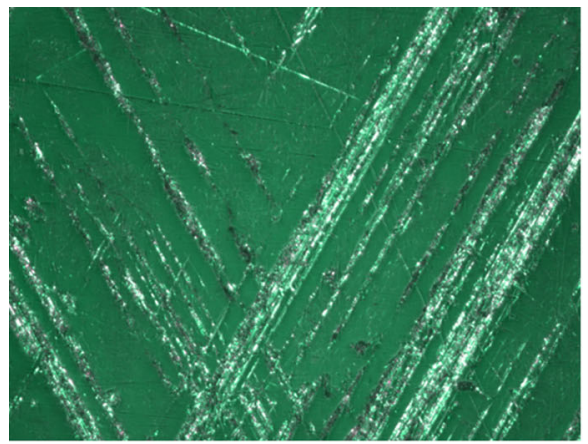

(a)

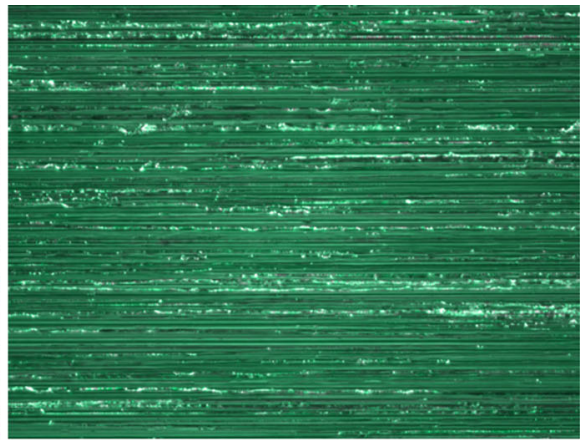

(b)

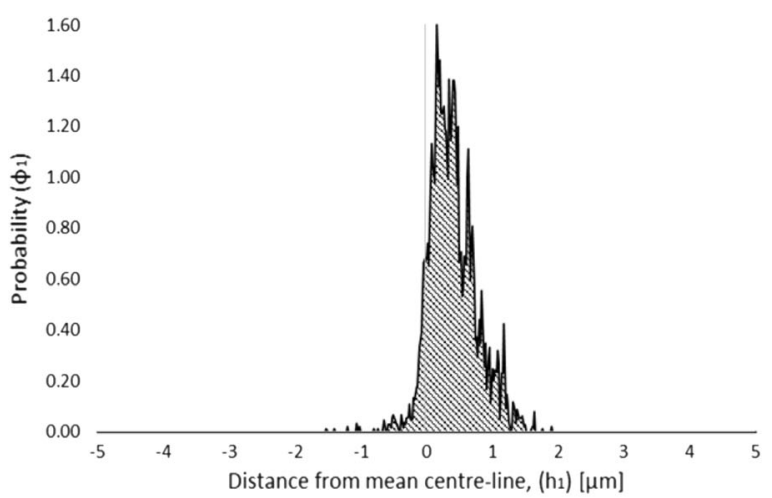

(a)

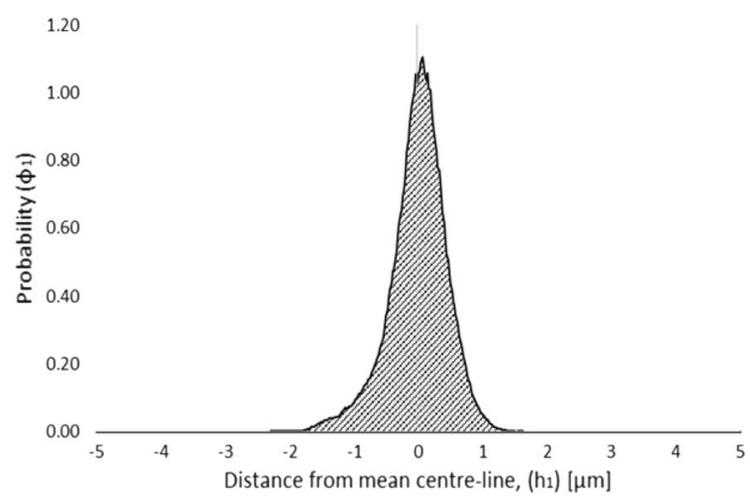

(b)

Fig. 3 Characteristic distributions for surface 1 averaged from 10 measured areas. a Peak height distribution. b Surface height distribution

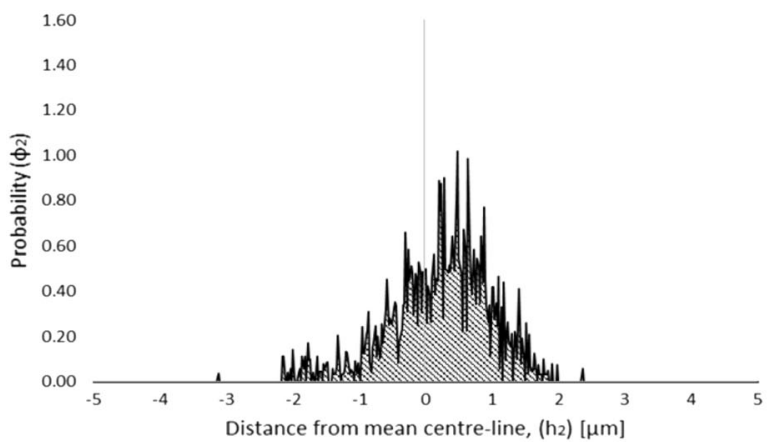

(a)

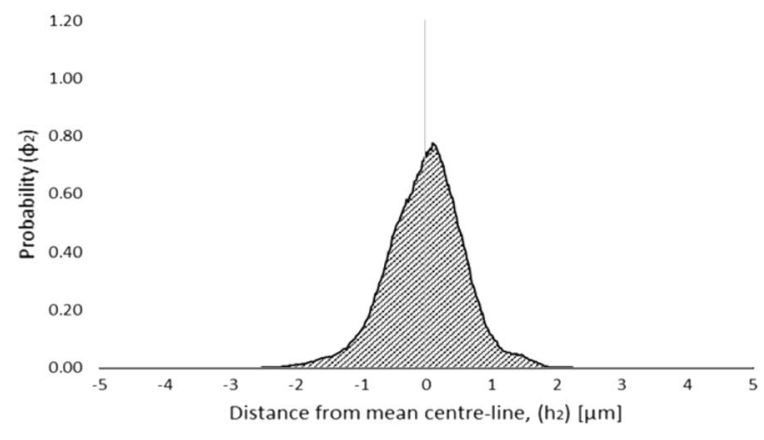

(b)

Fig. 4 Characteristic distributions for surface 2 averaged from 10 measured areas. a Peak height distribution. b Surface height distribution

roughness similar to that of a piston ring (surface 2 as shown in Fig. 2b). The peak and surface height distributions for these surfaces are shown in Figs. 3 and 4.

The convoluted distributions for the pairs of contacting surfaces 1 and 2 are shown in Fig. 5 .
It can be seen from the convolution of the peak height distributions (Fig. 5a) that there are significantly more asperities above the centre-line than would be predicted by a Gaussian distribution with a mean of 0 . Not only is there a non-Gaussian peak height distribution, but also the mean height is non- 


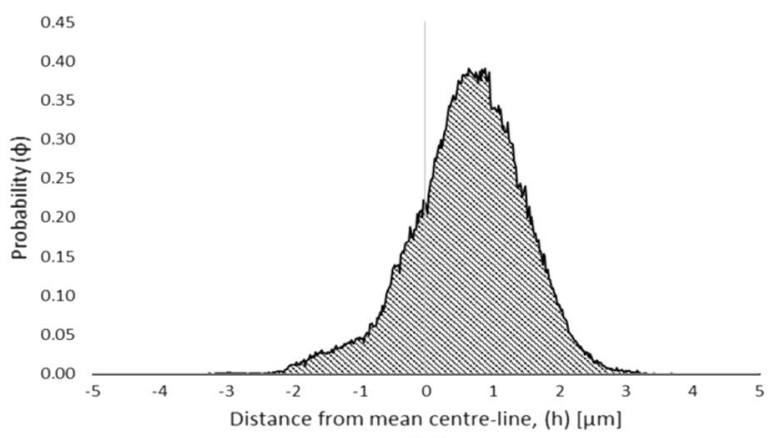

(a)

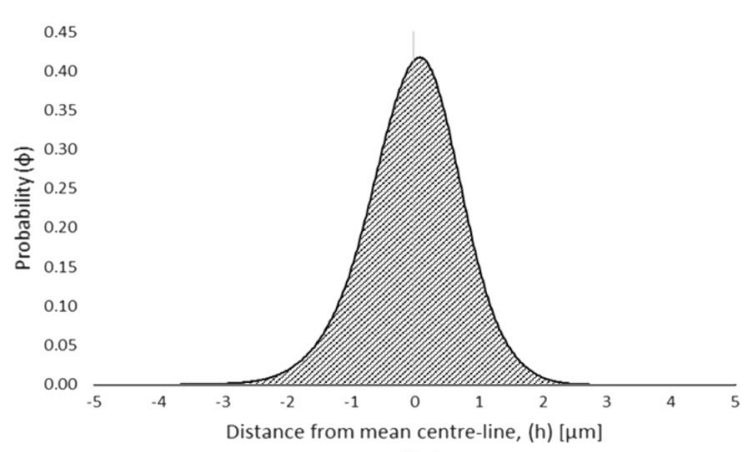

(b)

Fig. 5 Convoluted characteristic distributions for contacting pairs averaged from 10 measured areas. a Peak height distribution. b Surface height distribution

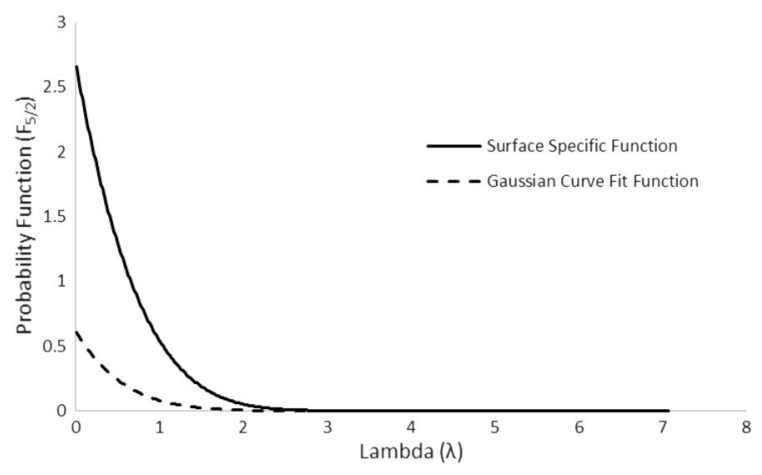

Fig. 6 Comparison between $F_{5 / 2}$ functions from surface specific data and from an assumed Gaussian distribution

zero which can also be seen for surface 2 with approximately Gaussian peak height and surface height distributions. This was given in the curve fit process of Greenwood and Tripp [10] or subsequent studies which have used this distribution assumption.

A comparison between the surface specific $F_{5 / 2}(\lambda)$ function and the same function provided by Greenwood and Tripp [10] for a Gaussian surface distribution, to which Arcoumanis et al. [23] fitted a 6th order curve fit, is shown in Fig. 6.

Figure 6 shows significant differences between the calculated functions. This is in part due to the increased proportion of asperities in the higher regions of the surface for the case of surface specific data. There is also a greater cumulative effect in these differences as the surfaces are moved together and the asperities continue to deform to a greater extent. For this case, the Gaussian assumption under predicts the $F_{5 / 2}$ function for these surfaces by over $80 \%$.
Figure 7 shows the mean asperity radius, $\beta_{i}(h)$, above a certain height as measured from the mean centre-line of the surface topography.

It is clear from Fig. 7 that a mean value used for contacting asperity radii, $\beta$, would not be a fair representation. Therefore, a more complex analysis of asperity radii is required. Determining $\beta(h)$ as a variable for the combination of the two surfaces under consideration gives the curve shown in Fig. 8 as the convolution of the asperity radii distributions for the rough counter surfaces.

Figure 8 shows a significant variation in $\beta(h)$ at the level of the upper asperities even though surface 2 has a minimal radius variation (Fig. 7b). The characteristic equations for asperity load share (Eq. 14) and contact area (Eq. 15) show that the variation in the asperity radius with mutually converging surfaces would significantly alter the load carrying capacity and thus any predicted generated friction. To apply the variables $\beta(h)$ and $\Psi(h)$, sixth order polynomial curve fits are used in the numerical model. The full range of the surface height distributions has bee shown in Figs. 7 and 8 to demonstrate the convergence to the mean. On the other hand, the Greenwood and Tripp model [10] is concerned only with the upper reaches of the topography.

\section{Validation of methology}

The methodology for representation of contact of a pair of rough surfaces (described above) using surface specific data is validated against experimental 
Fig. 7 Asperity radius distributions for the counter surfaces 1 and 2, averaged from 10 measured areas. a Surface 1 . b Surface 2
Fig. 8 Convoluted asperity radius and curvature distributions averaged from 10 measured areas
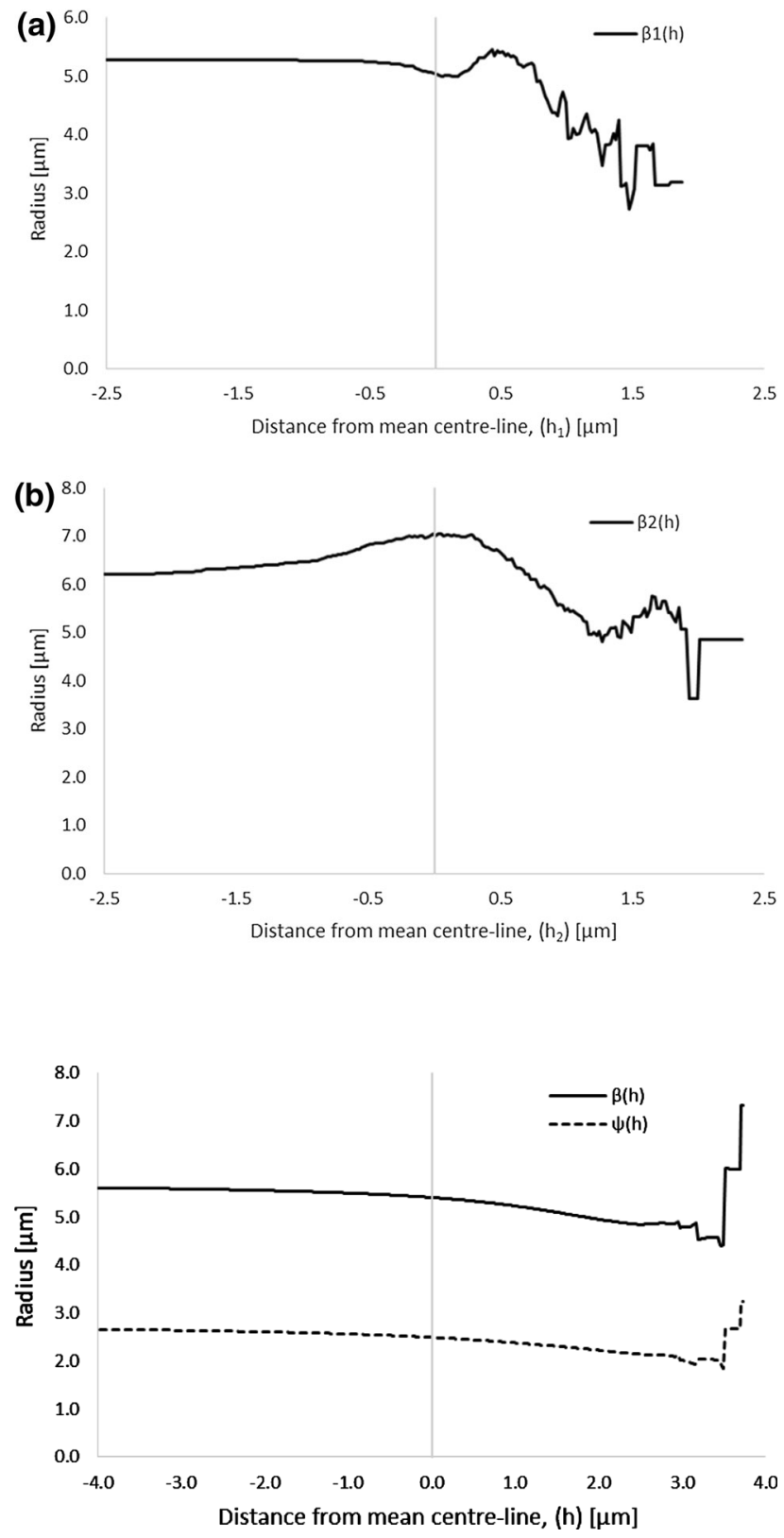

measurements of friction from a precision sliding tribometer.

\subsection{Sliding tribometer}

A reciprocating slider tribometer is used to measure the generated friction between the two measured rough surfaces. A 'strip' (surface 2 described above) comprising a $20 \times 20 \mathrm{~mm}$ flat area with $45^{\circ}$ chamfered inlet and outlets slides on a flat sample (surface 1 described above), the latter mounted onto a floating flat plate, supported by frictionless bearings and intervened from the solid base of the rig by piezoresistive load cells (Fig. 9). The floating plate is dragged by the sliding contact conjunction, lubricated by $1 \mathrm{ml}$ of base oil applied to the plate surface, furnishing a very thin lubricant film and significant asperity interactions. The drag force (generated 
Fig. 9 Detail view of the sliding tribometer

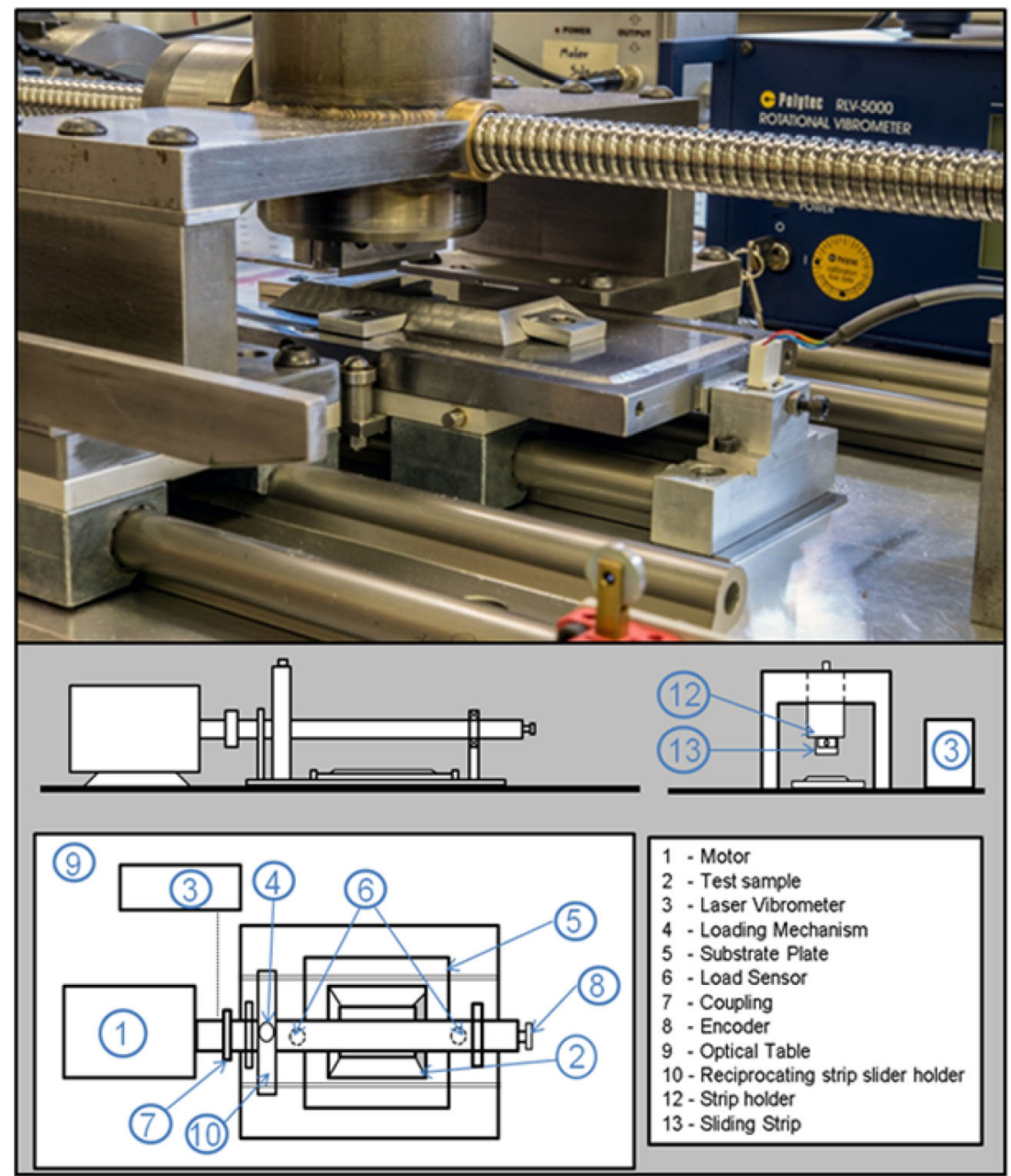

\begin{tabular}{lll}
\hline Parameter & Value & Unit \\
\hline Eyring shear stress $\left(\tau_{o}\right)$ & 2 & $\mathrm{MPa}$ \\
Lubricant density $(\rho)$ & $839.3\left(\right.$ at $\left.20^{\circ} \mathrm{C}\right)$ & $\mathrm{Kg} / \mathrm{m}^{3}$ \\
Lubricant dynamic viscosity $\left(\eta_{0}\right)$ & $0.1583\left(\right.$ at $\left.20^{\circ} \mathrm{C}\right)$ & $\times 10^{-6} \mathrm{~Pa} \mathrm{~s}$ \\
\hline
\end{tabular}

Table 1 Lubricant data

The pertinent base oil rheological parameters are listed in Table 1.

The rig is operated at low sliding velocity, representative of poor kinematic conditions of piston motion near the dead centre reversals and in this case under isothermal conditions and at relatively low load. The load is applied through the loading mechanism shown in the figure. Mixed or boundary regimes of lubrication would be expected under these conditions. Further details about the slider rig and its operation are provided in Morris et al. [24]. 
Relatively low load and large apparent contact area, $\mathcal{A}$ (contacting face of the strip) yields insufficient lubricant pressures to cause any elasto-hydrodynamic deformation of the surfaces. Furthermore, any lubricant film thickness, $h$, above the mean surface height of topography, $d$, is expected to be quite thin, yielding a mixed regime of lubrication.

\subsection{The numerical model}

The generated friction under the anticipated mixedhydrodynamic regime of lubrication is obtained as:

$f=f_{v}+f_{b}$

where, the total friction, $f$ is as the result of contributions due to the viscous shear of a thin lubricant film and boundary friction due to the interaction of asperities on the counterfaces of the contacting surfaces.

The viscous friction force is obtained as follow:

$f_{v}=\int_{0}^{L} \int_{-b / 2}^{b / 2}\left| \pm \frac{h}{2} \vec{\nabla} p-U \frac{\eta}{h}\right| d x d y$

Boundary friction is due to the interaction of counterface asperities, as well as any pockets of lubricant entrapped between them, which are assumed to be subject to the limiting Eyring [25] shear stress as [9]:

$f_{b}=\tau_{0} \tilde{A}+\varsigma \tilde{P}$

where, $\tilde{A}$ is given by Eq. (15). This value is clearly different for the measured surface specific topography and that based on the assumption of Gaussian distribution of Greenwood and Tripp [10] model. Therefore, the resultant friction predicted for an assumed Gaussian peak height distribution, using Greenwood and Tripp [10] model and that for peak height distribution determined using the measured surface data are compared with the directly measured friction using the experimental rig.

The first term on the right-hand side of Eq. (28) represents the non-Newtonian shear of thin pockets of lubricant. The second term corresponds to the direct interaction of asperities. $\varsigma$ is the coefficient of shear strength of asperities, measured using an atomic force microscope in the lateral force mode [8]. The Eyring shear stress is given in Table 1 , and $\varsigma=0.17$ [26].
It is clear that the lubricant film thickness, $h$ is required in order to predict the viscous friction contribution in Eq. (27). Therefore, a quasi-static load balance must be sought between the contact load carrying capacity, comprising asperity load share and any hydrodynamic lubricant reaction against the applied load, thus:

$W=W_{h y}+\tilde{P}(d)$

The hydrodynamic lubricant film reaction is obtained as:

$W_{h y}=\iint p d x d y$

The hydrodynamic generated pressure distribution is obtained through solution of Reynolds equation:

$\frac{\partial}{\partial x}\left(\frac{\rho h^{3}}{6 \eta} \frac{\partial p}{\partial x}\right)+\frac{\partial}{\partial y}\left(\frac{\rho h^{3}}{6 \eta} \frac{\partial p}{\partial y}\right)=U \frac{\partial(\rho h)}{\partial x}+2 \frac{\partial(\rho h)}{\partial t}$

where, $U$ is the sliding velocity of the strip in the $x$ direction. The strip is of finite length, thus the Poiseuille side-leakage flow due to pressure gradient in the lateral $y$-direction is also taken into account. The final term on the right-hand side of the equation takes the squeeze film effect into account due to the transient nature of the problem.

The lubricant film shape is given as:

$h(x, y)=h_{0}+\frac{x^{2}}{2 R}+\delta(x, y)$

where, $h_{0}$ is the initial gap. As already noted there is no localised elastohydrodynamic deformation of the contacting surfaces for the relatively low generated pressures, thus: $\delta(x, y) \cong 0$.

The boundary conditions used for the solution of Reynolds equation are:

$p_{(x=-b / 2)}=P_{a t m}, p_{(x=c)}=P_{c a v}$ and $\left.\frac{\partial p}{\partial x}\right|_{(x=c)}=0$

These correspond to atmospheric pressure at the inlet $(x=-b / 2)$, where $b$ is the effective face-width of the sliding strip and Swift-Stieber boundary conditions at the film rupture point at the contact exit constriction $(x=c)$, where the cavitation pressure is that of the lubricant at the environmental temperature 
Fig. 10 Comparison of friction predicted by various asperity models with experimental data

\author{
Experimentally Obtained Friction Measurement
}

Numerical Lubricant Model with Modified Asperity Model (Variable $\beta(\lambda)$ and $\psi(\lambda)$ ) and Surface Specific Probability Distributions

Numerical Lubricant Model with Modified Asperity Model (Constant $\beta$ and $\psi$ ) and Surface Specific Probability Distributions

Numerical Lubricant Model with Original Asperity Model [10] (Constant $\beta$ ) and Gaussian Probability Distributions
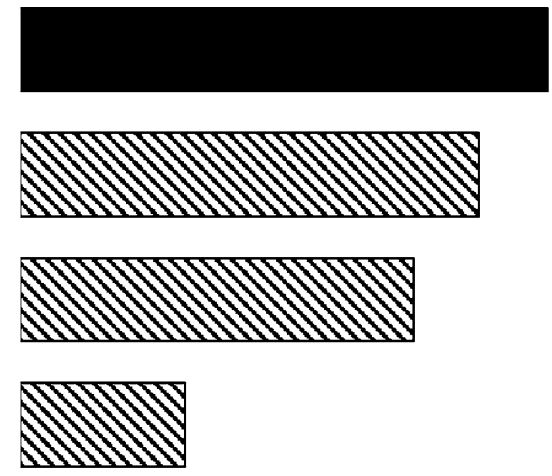

2.0

$\begin{array}{cc}2.4 & 2.6 \\ \text { Friction }[\mathrm{N}] & \end{array}$ 2.8

of $20{ }^{\circ} \mathrm{C}$. In this study the cavitation pressure is assumed to be equal to the atmospheric pressure.

In the lateral direction (side-leakage direction):

$p_{(y=0)}=p_{(y=l)}=P_{a t m}$

The rheological properties of the lubricant vary with pressure in the current isothermal analysis. The relationships given by Dowson and Higginson [27] and Houpert [28] are utilised to take into account the variations of lubricant density and dynamic viscosity with pressure and temperature as:

$\rho=\rho_{0}\left[1+\frac{6 \times 10^{-10}\left(p-p_{\text {atm }}\right)}{1+1.7 \times 10^{-9}\left(p-p_{\text {atm }}\right)}\right]$

and:

$$
\begin{aligned}
\eta= & \eta_{0} \exp \left\{\left(\ln \eta_{0}+9.67\right)\right. \\
& \left.\left(\left[1+5.1 \times 10^{-9}\left(p-p_{a t m}\right)\right]^{Z}-1\right)\right\}
\end{aligned}
$$

where:

$$
Z=\frac{\alpha}{5.1 \times 10^{-9}\left(\ln \eta_{0}+9.67\right)}
$$

A second order finite difference method is used to solve Reynolds equation by utilising Point-Successive Over-Relaxation scheme. During the iterations the lubricant properties are also updated. The procedure used, including for convergence criteria, are described in Rahmani et al. [29].

\section{Results and discussion}

Figure 10 shows a comparison of numerical predictions of friction with that measured using the sliding tribometer. In general, the numerical predictions show very good agreement with the measured data, with a maximum deviation from the measured friction of $3.98 \%$ in the case of boundary friction contribution, based on the Greenwood and Tripp model with a constant average asperity tip radius. This difference is further reduced to $2.67 \%$ when the boundary friction contribution is based on the surface-specific distribution data $\left(F_{5 / 2}\right.$ and $F_{2}$, thus $\tilde{P}(d)$ and $\left.\tilde{A}\right)$ with an assumed constant average asperity tip radius. An even further reduced difference (i.e. $2.47 \%$ ) from measured friction is obtained when surface-specific topographic distributions are used with a variable determined asperity tip radius $\beta(h)$ for any given separation $h$. Therefore, the results illustrate the importance of considering the asperity radii as a variable with respect to separation (i.e. $\beta(h)$ ). The progressive improved predictions account for small gains in accuracy, but represent significant gains in practice.

\section{Conclusions}

The current analysis highlights the potential misrepresentation which may occur as a result of assuming a Gaussian surface roughness distribution in modelling asperity interactions. Considering real surface-specific roughness distributions and comparing with experimental data the potential difference is clearly demonstrated. The repercussions of this for asperity load carrying capacity and also the onset of mixed regime of lubrication is described. 
It is also clear that the method of considering variation in asperity radii at different separations, developed here, offers additional improvements for surface analysis and modelling. The use of measured surface-specific distribution and separation-dependent variable asperity radii are the main contributions made to knowledge in this paper. The developed methodology also shows very good agreement with experimental measurements of friction.

Acknowledgments The authors would like to thank the UK Engineering and Physical Sciences Research Council (EPSRC) for the sponsorship of this research under the Encyclopaedic Program Grant (www.encyclopaedic.org).

Open Access This article is distributed under the terms of the Creative Commons Attribution 4.0 International License (http:// creativecommons.org/licenses/by/4.0/), which permits unrestricted use, distribution, and reproduction in any medium, provided you give appropriate credit to the original author(s) and the source, provide a link to the Creative Commons license, and indicate if changes were made.

\section{References}

1. Dowson D (ed) (1982) Tribology of reciprocating engines. In: Proceedings of 9th Leeds-Lyon symposium on tribology, 1982

2. Balakrishnan S, Rahnejat H (2005) Isothermal transient analysis of piston skirt-to-cylinder wall contacts under combined axial, lateral and tilting motion. J Phys D Appl Phys 38(5):787

3. Tipei N (1968) Boundary conditions of a viscous flow between surfaces with rolling and sliding motion. Trans ASME J Tribol 90(1):254-261

4. Shahmohamadi H, Mohammadpour M, Rahmani R, Rahnejat H, Garner CP, Howell-Smith S (2015) On the boundary conditions in multi-phase flow through the piston ring-cylinder liner conjunction. Tribol Int 90:164-174

5. Furuhama S, Sasaki S (1983) New device for the measurement of piston frictional forces in small engines. SAE technical paper, no. 831284, 1983

6. Bolander NW, Steenwyk BD, Sadeghi F, Gerber GR (2005) Lubrication regime transitions at the piston ring-cylinder liner interface. Proc IMechE Part J J Eng Tribol 219(1): 19-31

7. Gore M, Theaker M, Howell-Smith S, Rahnejat H, King PD (2014) Direct measurement of piston friction of internalcombustion engines using the floating-liner principle. Proc IMechE Part D J Automob Eng 228(3):344-354

8. Styles G, Rahmani R, Rahnejat H, Fitzsimons B (2014) Incycle and life-time friction transience in piston ring-liner conjunction under mixed regime of lubrication. Int J Engine Res 15(7):862-876
9. Teodorescu M, Kushwaha M, Rahnejat H, Rothberg SJ (2007) Multi-physics analysis of valve train systems: from system level to microscale interactions. Proc IMechE Part K J Multi Body Dyn 221(3):349-361

10. Greenwood J, Tripp J (1970) Contact of two nominally flat rough surfaces. Proc IMechE J Mech Eng Sci 185:625-633

11. Greenwood J, Williamson JBP (1966) Contact of nominally flat surfaces. Proc R Soc 295:300-319

12. Hisakado T (1974) Effect of surface roughness on contact between solid surfaces. Wear 28(2):217-234

13. Bush AW, Gibson RD, Keogh GP (1976) Strongly anisotropic rough surfaces. Trans ASME J Tribol 101(Part 1): $15-20$

14. McCool JI (1986) Comparison of models for the contact of rough surfaces. Wear 107(1):37-60

15. Pullen J, Williamson JBP (1972) On the plastic contact of rough surfaces. Proc R Soc 327(1569):159-173

16. Cheng HSA (1970) Numerical solution of the elastohydrodynamic film thickness in an elliptical contact. Trans ASME J Tribol 92(Part 1):155-161

17. Chong WWF, Teodorescu M, Rahnejat H (2013) Nanoscale elastoplastic adhesion of wet asperities. Proc IMechE Part J J Eng Tribol 227(9):996-1010

18. Ma Z, Henin A, Bryzik W (1997) A model for wear and friction in cylinder liners and piston rings. Tribol Trans 49(3):315-327

19. Mishra PC, Rahnejat H, King PD (2009) Tribology of the ring-bore conjunction subject to a mixed regime of lubrication. Proc IMechE Part C J Mech Eng Sci 223(4):987-998

20. Liu G, Wang Q, Lin C (2006) A survey of current models for simulating the contact between rough surfaces. Tribol Trans 42(3):581-591

21. Teodorescu M, Kushwaha M, Rahnejat H, Taraza D (2005) Elastodynamic transient analysis of a four-cylinder valvetrain system with camshaft flexibility. Proc IMechE Part K J Multi Body Dyn 219:13-25

22. Peklenik J (1967) Paper 24: new developments in surface characterization and measurements by means of random process analysis. Proc IMechE Conf 182(11)

23. Arcoumanis C, Ostovar P, Mortier R (1997) Mixed lubrication modelling of Newtonian and shear thinning liquids in a piston-ring configuration. In: SAE technical paper, 1997, pap no. 972924:35

24. Morris N, Leighton M, De la Cruz M, Rahmani R, Rahnejat H, Howell-Smith S (2015) Combined numerical and experimental investigation of the micro-hydrodynamics of chevron-based textured patterns influencing conjunctional friction of sliding contacts. Proc IMechE Part J J Eng Tribol 229(4):316-335

25. Eyring H (1936) Viscosity, plasticity, and diffusion as examples of absolute reaction rates. J Chem Phys 4(4): 283-291

26. De la Cruz M, Chong WWF, Teodorescu M, Theodossiades S, Rahnejat H (2012) Transient mixed thermo-elastohydrodynamic lubrication in multi-speed transmissions. Tribol Int 49:17-29

27. Dowson D, Higginson GR (1959) A numerical solution to the elasto-hydrodynamic problem. J Mech Eng Sci 1(1): 6-15 
28. Houpert L (1985) New results of traction force calculations in elastohydrodynamic contacts. Trans ASME J Tribol 107(2):241-245

29. Rahmani R, Theodossiades S, Rahnejat H, Fitzsimons B (2012) "Transient elastohydrodynamic lubrication of rough new or worn piston compression ring conjunction with an out-of-round cylinder bore. Proc IMechE Part J J Eng Tribol 226(4):284-305 